\title{
Relationships of Direction and Degree of Nasal Septum Deviation with Nasal Floor Morphology
}

\author{
Soo Kweon Koo, MD, PhD, Geun Hyung Park, MD, Tae Kyung Koh, MD, PhD, Ho Byung Lee, MD \\ and Chang Lok Ji, MD \\ Department of Otorhinolaryngology-Head and Neck Surgery, Busan Saint Mary's Hospital, Busan, Korea
}

\begin{abstract}
- ABSTRACT -
Background and Objectives: Nasal septum deviation can affect nasal bone growth and facial morphology. We used computed tomography image to clarify the relationships between nasal septal deviation and nasal floor morphology in Koreans. Materials and Methods: From 2006 to 2016, CT images of 687 patients of nasal septal deviation and 100 of non-nasal septal deviation were retrospectively analyzed. Relationship between nasal septum and nasal floor was analyzed on 271 subjects whose nasal septum and nasal floor are both deviated. Only cephalocaudal C-shape was analysed, based on the Guyuron classification. The angle of the nasal floor measured at three vertical reference planes were used to assess the relationships with angle of nasal septal deviation. Results: The number of patients with nasal floor deviation was $39.4 \%$ in nasal septal deviation group and $17.0 \%$ in control group, respectively; nasal floor was more uneven when nasal septum deviated $(\mathrm{p}<0.001)$. In 271 patients with both nasal septum and floor deviation, the mean angles of nasal septum and nasal floor were $11.24^{\circ}$ and $5.60^{\circ}$ respectively. The direction of nasal floor was significantly deviated in the opposite to nasal septum $(28 \%: 72 \%, \mathrm{p}<0.01)$ in both sex. But the degree of asymmetry of the nasal floor was not significantly correlated with the degree of nasal septal deviation. Conclusions: Nasal floor asymmetry occurred in the opposite direction to nasal septal deviation in $72 \%$ of patients. In patients with nasal septal deviation during growth, facial growth may occur asymmetrically, which is an important consideration during treatment of nasal septal deviation. (J Clinical Otolaryngol 2020;31:181-187)
\end{abstract}

KEY WORDS: Nasal septum · Facial bones · Asymmetry · Computed tomography.

\section{Introduction}

The nasal septum is located in the midline of the nasal cavity and is an important component of the nasal structure. Anatomically, the anterior half of the nasal septum consists of the quadrangular cartilage; the posterior half consists of the vomer and the perpendicular plate of the ethmoid bone. ${ }^{1,2)}$ Although the incidence of nasal septal deviation differs among regions, it is the most common nasal anatomical finding in healthy adults and has been reported in up to $80 \%$ of individu- als in a given population. ${ }^{3,4)}$ Nasal septal deviation plays an important role in nasal congestion symptoms, nasal aesthetic appearance, increased nasal resistance, and occasional snoring. ${ }^{5)}$ Thus, evaluation of the nasal septum is important for pre-surgical planning, reconstruction, and overall cosmetic results.

Although there is some controversy, nasal septal deviation has been shown to affect nasal bone growth and facial morphology. From the early stages of growth, the maxilla and nasal structures have close anatomical connections, due to the close relationships of these struc-

논문접수일: 2020년 9월 15일 / 논문수정일: 2020년 11월 11일 / 심사완료일: 2020년 12월 11일

교신저자: 구수권, 48575 부산광역시 남구 용호로 232번길 25-14(용호동), 부산성모병원 이비인후과

전화: (051) 933-7214 · 전송: (051) 956-1956·E-mail: koosookweon@ naver.com 
tures during embryonic development. ${ }^{1-4,6)}$ During the growth stage, the nasal septum acts as a growth plate that affects the surrounding bone and facial skeletal tissue. ${ }^{7)}$ Therefore, nasal septal deviation greatly affects the morphological structure of the face; understanding the parameters of this deviation plays an important role in septoplasty and rhinoplasty.

Thus far, few studies have evaluated the correlation between the facial skeletal structure and nasal septal deviation using computed tomography (CT) scans. The shapes of the facial bones vary widely, according to sex, age, region, and ethnicity. From this perspective, this study of Korean patients provides important information. We used CT imaging to clarify the relationships between intranasal structures (i.e., nasal floor type) and nasal septal deviation in Koreans with nasal septal deviation.

\section{Materials and Methods}

From June 2006 to June 2016, CT images of 687
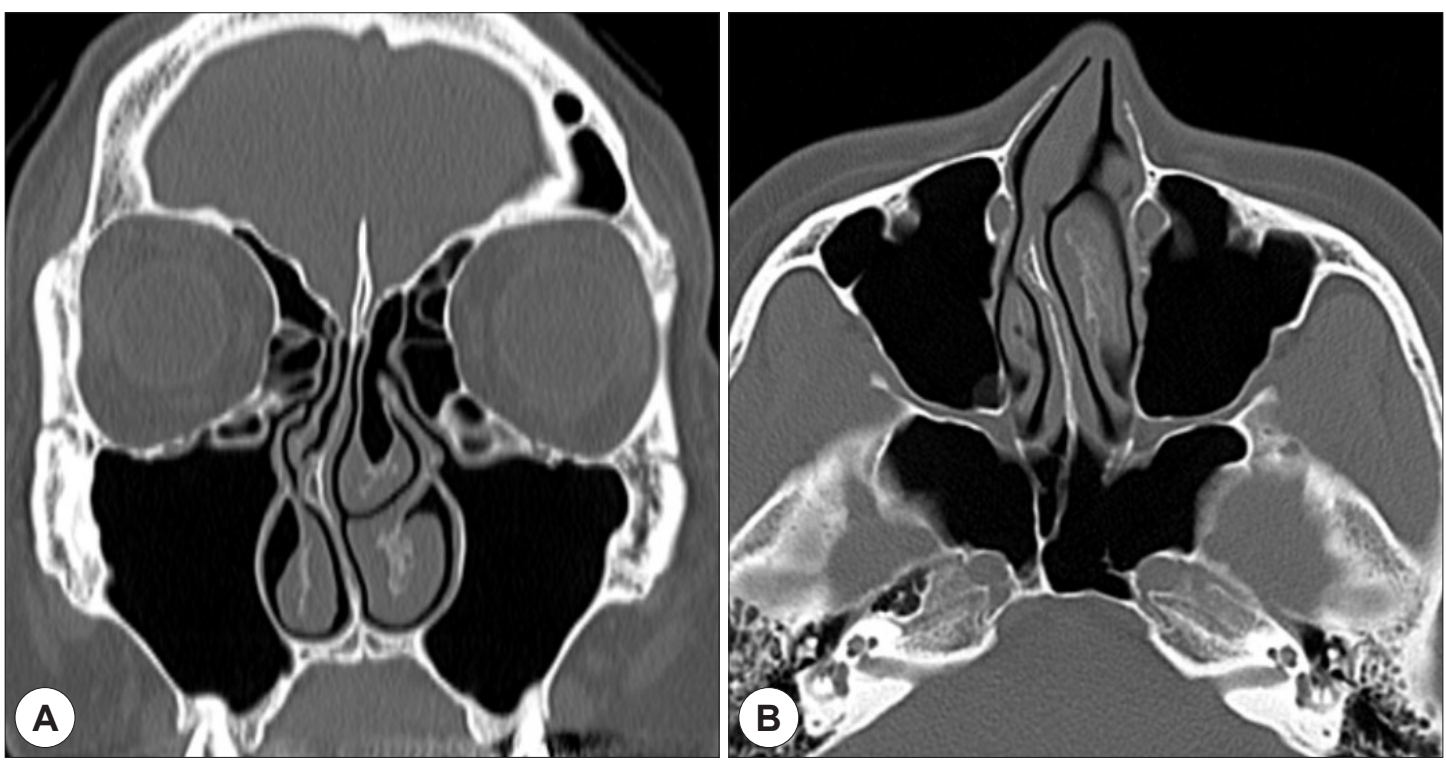

Fig. 1. CT image of cephalocaudal C type deviation, according to Guyuron classification. A : Coronal view, B : Axial view. patients of nasal septal deviation and 100 of non-nasal septal deviation were retrospectively analyzed. 271 of 687 patients (221 men, 50 women) with both nasal septal deviation and nasal floor deviation were included for study group to evaluate statistical correlation of the two nasal structures. The average ages were 29.08 years in men and 30.66 years in women. Patients with a history of rhinoplasty, skull and facial trauma or bone deformity, and/or intranasal sinus lesions, were excluded from the study. The analysis was performed by one otolaryngologist to prevent inter-examiner errors.

In nasal septal deviation group, only cephalocaudal $\mathrm{C}$-shape based on the Guyuron classification was analyzed to exclude bias (Fig. 1). ${ }^{8)}$ The angle of the nasal floor measured in each three vertical reference planes at internal nasal landmarks, which were defined as the anterior nasal spine, the central point of the nasal floor (50\% of the distance from the anterior nasal spine to the posterior nasal spine), and the posterior nasal spine (Fig. 2). The angles of the nasal floor were defined as the angles between the lowest nasal floors of both nasal 


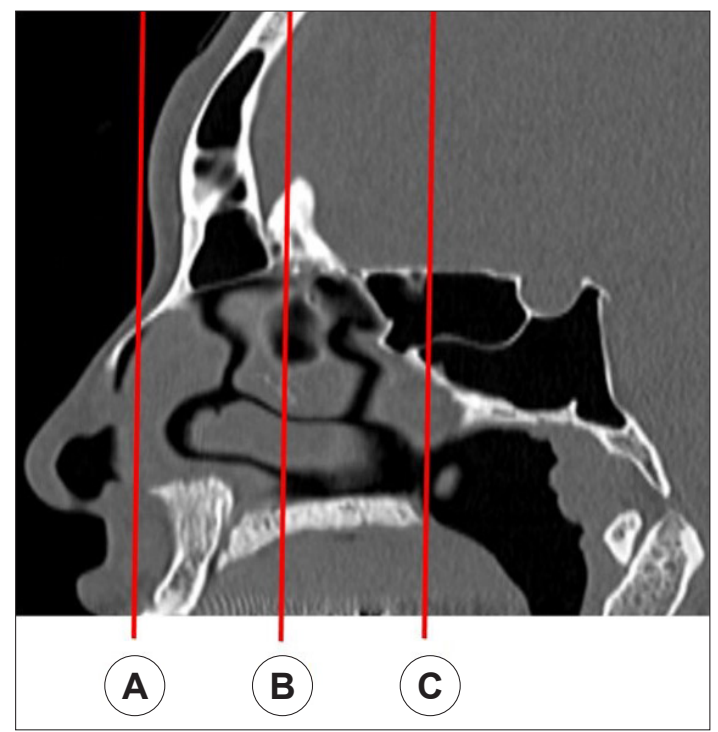

Fig. 2. Three vertical reference planes. A : anterior nasal spine, B : $50 \%$ of distance from anterior nasal spine to posterior nasal spine, $\mathrm{C}$ : posterior nasal spine.

cavities in the same plane (Fig. 3). The angles of septal deviation were defined as the angle between the most deviated point and the midline in coronal $\mathrm{CT}$ images and the direction of deviation (left, right) were descript- ed (Fig. 3).

CT examination was performed using an Activion 16 CT scanner (Toshiba Medical Systems, Tokyo, Japan). CT parameters were $120 \mathrm{kVp}, 100-150 \mathrm{~mA}, 0.5 \mathrm{~mm}$ adjacent axial slice thickness, $512 \times 512$ matrix size, and field of view 240. Images were obtained with patients in the supine position without rotation, flexion or expansion; they were analysed using axial and coronal views.

Our study adhered to the tenets of the 2013 Declaration of Helsinki; the study protocol was approved by the Ethics Committee of Busan Saint Mary's Hospital (approval number BSM2020-02).

All statistical analyses were performed using SPSS Statistics for Windows (ver. 18.0; SPSS, Inc., Chicago, IL, USA). To assess the relationship between the direction of nasal septal deviation and the direction of nasal floor asymmetry, patients were numbered in the same or opposite directions; the chi-squared test was used to compare proportions between these groups. The Pearson correlation coefficient was used to examine the relationship between the degree of septal deviation and the degree of nasal floor deviation.
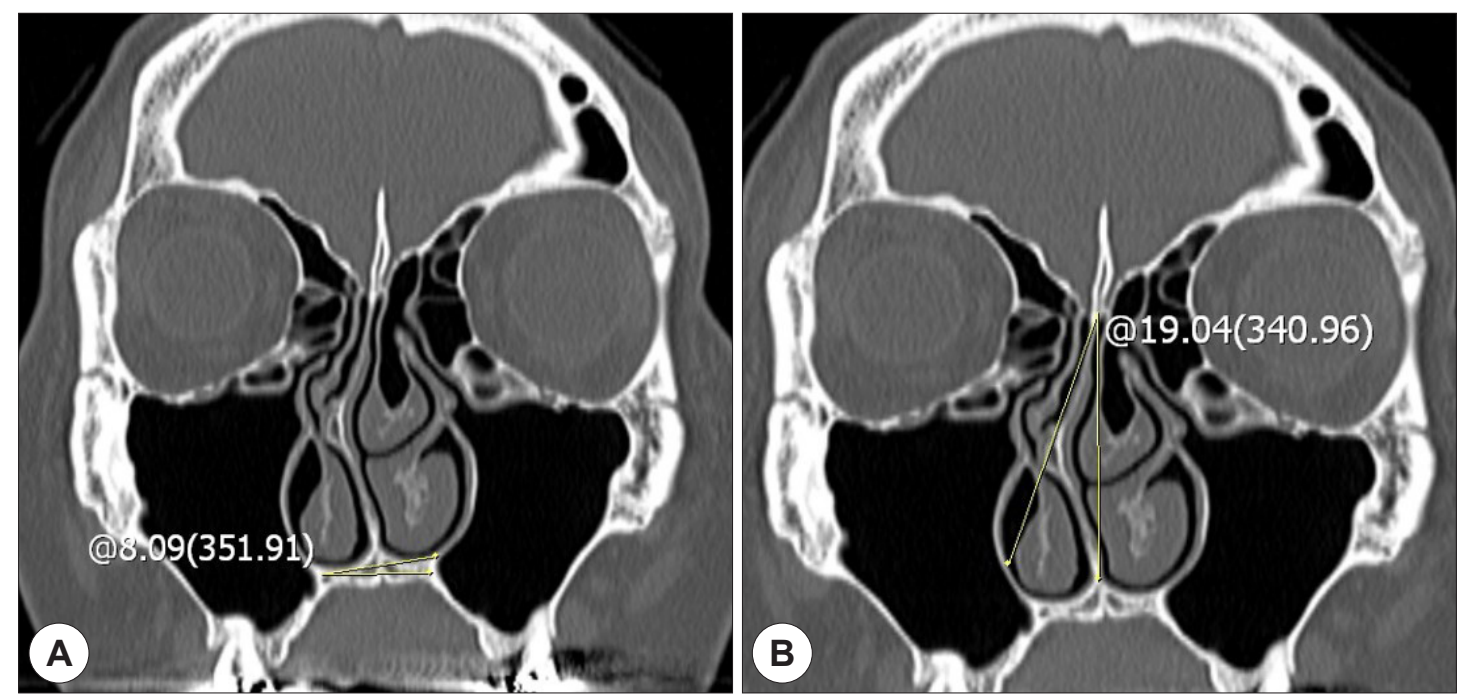

Fig. 3. The angle of nasal floor. (A) angle of nasal floor (angle between lowest nasal floors of both nasal cavities in same plane), (B) angle of septal deviation (angle between most deviated point and midline). 


\section{Results}

The number of patients with nasal floor deviation was 271/687 (39.4\%) in nasal septal deviation group, and $17 / 100(17.0 \%)$ in control group, respectively, so there was tendency of nasal floor to be more uneven when there was nasal septal deviation $(\mathrm{p}<0.001)$ and 271 were finally included for study group (Table 1).

\section{Patient characteristics and mean degrees of} septal and nasal floor deviation

The direction of nasal septal deviation was 6:4, more often deviated to the left; this was consistent in both men and women. The angles of septum deviation were $11.00^{\circ}$ in men and $12.31^{\circ}$ in women; deviation was sig- nificantly more severe in women $(\mathrm{p}=0.034)$. The angles of the nasal floor (anterior, middle, posterior) were $7.04^{\circ}$, $5.92^{\circ}$ and $3.79^{\circ}$ in men and $7.46^{\circ}, 6.19^{\circ}$ and $3.48^{\circ}$ in women; no significant differences were found between men and women (Table 2).

\section{Relationships between direction of nasal septal deviation and direction of nasal floor asymmetry}

The asymmetry (deviation) of the nasal floor was significantly deviated in the opposite direction: $28 \%$ in the direction of nasal septal deviation and $72 \%$ in the opposite direction $(p<0.01)$. This relationship also appeared in each sex $(p<0.01)$ (Table 3$)$ and was shown in schematic diagram (Fig. 4).

Table 1. Ratio of patients with nasal floor deviation in septal deviation group $(n=687)$ and non-septal deviation group $(\mathrm{n}=100)$ (control group)

\begin{tabular}{lccc}
\hline & Septal deviation group & Non-septal deviation group & P value \\
\hline Nasal floor deviation ratio & $39.4 \%(271 / 687)$ & $17.0 \%(17 / 100)$ & $<0.001$ \\
\hline
\end{tabular}

Table 2. Patient characteristics and mean degree of septal and nasal floor deviation

\begin{tabular}{|c|c|c|c|}
\hline & Male $(n=221)$ & Female $(n=50)$ & $P$ value \\
\hline Age (years) & $29.08 \pm 13.15$ & $30.66 \pm 13.82$ & 0.447 \\
\hline $\begin{array}{l}\text { Direction of septal deviation } \\
\text { (right : left) }\end{array}$ & $89: 132$ & $20: 30$ & \\
\hline Mean angle of Septal deviation $\left(^{\circ}\right)$ & $11.00 \pm 3.84$ & $12.31 \pm 4.11$ & 0.034 \\
\hline $\begin{array}{l}\text { Mean angle of anterior nasal floor } \\
\text { deviation }\left({ }^{\circ}\right)\end{array}$ & $7.04 \pm 4.13$ & $7.46 \pm 4.37$ & 0.527 \\
\hline $\begin{array}{l}\text { Mean angle of middle nasal floor } \\
\text { deviation }\left({ }^{\circ}\right)\end{array}$ & $5.92 \pm 3.41$ & $6.19 \pm 3.30$ & 0.617 \\
\hline $\begin{array}{l}\text { Mean angle of posterior nasal floor } \\
\text { deviation }\left(^{\circ}\right)\end{array}$ & $3.79 \pm 2.55$ & $3.48 \pm 2.44$ & 0.437 \\
\hline
\end{tabular}

Values of angle are presented with mean \pm standard deviation. Full angle is 360 degree.

Table 3. Frequency of the direction of nasal floor deviation according to the direction of nasal septal deviation

\begin{tabular}{lcccc}
\hline & *Same direction & TOpposite direction & Chi-square & P value \\
\hline Male & $66(29.9 \%)$ & $155(70.1 \%)$ & 35.842 & $<0.001$ \\
Female & $10(20.0 \%)$ & $40(80.0 \%)$ & 18.000 & $<0.001$ \\
\hline Total & $76(28.0 \%)$ & $195(72.0 \%)$ & 52.255 & $<0.001$
\end{tabular}

* Same direction: left septal deviation with left nasal floor deviation; right septal deviation with right nasal floor deviation. † Opposite direction: left septal deviation with right nasal floor deviation; right septal deviation with left nasal floor deviation. 


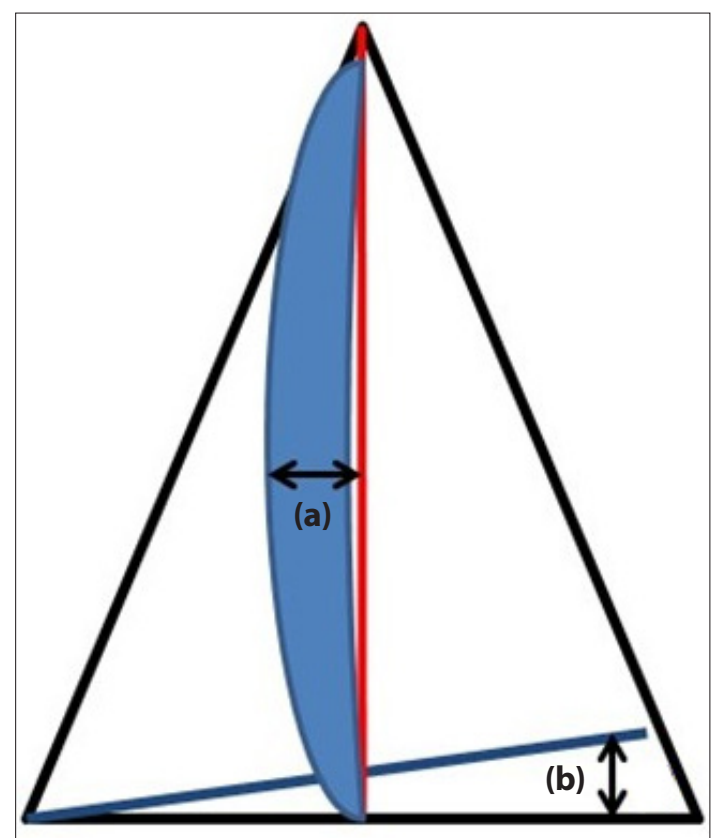

Fig. 4. Schematic diagram of relationship between nasal septal deviation and nasal floor. (a) angle of nasal septal deviation, (b) angle of nasal floor. In patients with nasal septal deviation, internal nasal parameters (angle of nasal floor) tended to be distorted in opposite direction from nasal septal deviation

\section{Correlation between degree of nasal floor asym-} metry and degree of nasal septal deviation

The degree of asymmetry of the nasal floor was not significantly correlated with the degree of nasal septal deviation in the same or opposite direction groups in the anterior, middle, and posterior planes (Table 4); the Pearson correlation coefficient was also not statistically significant in either sex group (Table 5).

\section{Discussion}

Many patients with nasal septum deviation are encountered in otorhinolaryngology practice; surgery to correct this condition is the most common and important operation in the treatment of nasal obstruction. In addition, most patients with nasal septal deviation display facial bone asymmetry; understanding this asymmetry is important for achievement of therapeutic success, such as in rhinoplasty or septoplasty.

To exclude bias related to the direction of nasal septal deviation, we targeted only patients with cephalocaudal $\mathrm{C}$-shaped deviation in accordance with the classification system proposed by Guyuron et al. ${ }^{8)}$ Guyuron et al. divided septal deviations into six classes, which included $\mathrm{C}$ - and S-shaped deviations in the anteroposterior and cephalocaudal directions, as well as localized deviation with a nasal spur and septal tilt. ${ }^{8)}$

The effect of the nasal septum on facial skeleton growth remains controversial. Some researchers have reported that the nasal septum plays a key role in the growth of the facial skeleton, while others consider it to play only a secondary role as support for facial development. ${ }^{7)}$ Hafezi et al. ${ }^{9}$ reported a significant delay in facial growth on the concave side of a deviated nose. Hartman et al. ${ }^{7)}$ revealed no correlation between septal deviation and lateral facial asymmetry as a result of multivariate regression analysis; however, there was a significant relationship between nasal septum deviation and local asymmetry in the nasal and palatal regions.

Table 4. Pearson correlation coefficient of degree of septal deviation with degree of nasal floor deviation at three vertical plane (anterior, middle, posterior)

\begin{tabular}{lcccc}
\hline & $\mathrm{n}$ & Anterior plane & Middle plane & Posterior plane \\
\hline *Same direction group & 76 & $-.011(.889)$ & $-.046(.562)$ & $-.039(.628)$ \\
†Opposite direction group & 195 & $.090(.064)$ & $.015(.755)$ & $.039(.422)$ \\
\hline Total & 271 & $.092(.130)$ & $-.045(.459)$ & $.003(.960)$ \\
\hline
\end{tabular}

Values are presented as Pearson correlation coefficient ( $p$ value). *Same direction: left septal deviation with left nasal floor deviation; right septal deviation with right nasal floor deviation. †Opposite direction: left septal deviation with right nasal floor deviation; right septal deviation with left nasal floor deviation. 
Table 5. Pearson correlations of degrees of septal deviation with degrees of nasal floor deviation in three vertical planes (anterior, middle, posterior), according to sex

\begin{tabular}{lcccc}
\hline & $\mathrm{n}$ & Anterior plane & Middle plane & Posterior plane \\
\hline Male & 221 & $.148(.028)$ & $-.046(.496)$ & $.019(.781)$ \\
Female & 50 & $-.150(.300)$ & $-.079(.584)$ & $-.033(.823)$ \\
\hline Total & 271 & $.092(.130)$ & $-.045(.459)$ & $.003(.960)$ \\
\hline
\end{tabular}

Values are presented as pearson correlation coefficient ( $p$ value).

Among the local asymmetries, nasal septum deviation was specifically associated with nasal floor asymmetry, consistent with our findings. In addition, the results of the present study that asymmetry of the nasal floor was in the opposite direction from the nasal septum curve was largely consistent with the findings of Hafezi et al., ${ }^{9}$ in that the development of the concave side of the nasal septum was delayed.

Several studies have shown that nasal septal hyperplasia affects facial asymmetry. Takahashi et al. ${ }^{10)}$ reported that when nasal septal hyperplasia occurs early in fetal development, it contributes to asymmetrical development of the palatal and nasal areas. Gray and Brogan, ${ }^{11,12}$ as well as other clinicians, have reported that nasal septal deviation occurring in the early stages is related to asymmetrical development of dental occlusion.

However, there remains controversy regarding how early nasal septal deviation affects facial bone growth. Because our study was conducted in adults, we could not clearly determine whether nasal septal deviation directly affects the fetal facial growth process; however, our findings suggest that nasal septal deviation is associated with asymmetry of the face after cessation of facial skeletal growth. If CT or photographic data could be used to determine the contour of the face before the development of nasal septal deviation, it may be possible to more clearly predict this relationship. Our study was retrospective in nature; thus, further research is needed.

Hartman et al. ${ }^{7)}$ reported that asymmetry of the nasal floor was evident in the posterior nasal cavity, partic- ularly compared to the anterior nasal cavity; however, our findings indicated that the anterior portion of the deviation was more severe. These conflicting results require further assessment through additional studies.

CT scans have not been extensively used to evaluate the relationships between facial asymmetry and nasal septal deviation in korean. Present study using CT images of patients undergoing surgery for septal deviation suggest the role of nasal septum development in facial bone formation.

In summary, nasal floor asymmetry occurred in the opposite direction from nasal septal deviation in approximately $70 \%$ of patients. However, the degree of asymmetry was not significantly correlated. In patients with nasal septal deviation during growth, facial growth may occur asymmetrically, which is an important consideration during treatment of nasal septal deviation.

\section{REFERENCES}

1) Kajan ZD, Khademi J, Nemati S, Niksolat E. The effects of septal deviation, concha bullosa, and their combination on the depth of posterior palatal arch in cone-beam computed tomography. J Dent 2016;17(1):26-31.

2) Wang J, Dou X, Liu D, Song P, Qian X, Wang S, et al. Assessment of the effect of deviated nasal septum on the structure of nasal cavity. Eur Arch Otorhinolaryngol 2016;273(6):1477-80.

3) Roblin D, Eccles R. What, if any, is the value of septal surgery? Clin Otolaryngol Allied Sci 2002;27(2):77-80.

4) Van Egmond M, Rovers M, Hendriks C, Van Heerbeek N. Effectiveness of septoplasty versus non-surgical management for nasal obstruction due to a deviated nasal septum in adults: study protocol for a randomized controlled trial. Trials 2015; 16:500.

5) Hsia JC, Camacho M, Capasso R. Snoring exclusively during nasal breathing: a newly described respiratory pattern during sleep. Sleep Breath 2014;18(1):159-64.

6) Ballanti F, Baldini A, Ranieri S, Nota A, Cozza P. Is there a 
correlation between nasal septum deviation and maxillary transversal deficiency? A retrospective study on prepubertal subjects. Int J Pediatr Otorhinolaryngol 2016;83:109-12.

7) Hartman C, Holton N, Miller S, Yokley T, Marshall S, Srinivasan $\mathrm{S}$, et al. Nasal septal deviation and facial skeletal asymmetries. Anat Rec 2016;299(3):295-306.

8) Guyuron B, Uzzo CD, Scull H. A practical classification of septonasal deviation and an effective guide to septal surgery. Plast Reconstr Surg 1999;104(7):2202-9.

9) Hafezi F, Naghibzadeh B, Nouhi A, Yavari P. Asymmetric facial growth and deviated nose: a new concept. Ann Plast Surg 2010;64(1):47-51.

10) Takahashi R. The formation of the nasal septum and the etiology of septal deformity: the concept of evolutionary paradose. Acta Otolaryngol Suppl 1987;443:1-160.

11) Gray LP, Brogan WF. Septal deformity malocclusion and rapid maxillary expansion. Orthodontist 1972;4(1):2-14.

12) Gray LP. The development and significance of septal and dental deformity from birth to eight years. Int J Pediatr Otorhinolaryngol 1983;6(3):265-77. 